\title{
Diseño metodológico para la estructuración de portafolios de inversión según el perfil de riesgo del inversionista
}

\author{
Methodological design for structuring investment portfolios according to the risk profile \\ of the investor
}

María Patricia Durango-Gutiérrez

Doctoranda en Ciencias Económicas y

Empresariales. Universidad de Granada

- España. Magister en Administración

Financiera Universidad EAFIT - Colombia.

Docente e investigadora de la Universidad

EAFIT, Medellín, Colombia.

Email: mdurango@eafit.edu.co

ORCID: https://orcid.org/0000-0001-8955-2706

Luis David Delgado-Vélez

Master en Finanzas y Contabilidad

Directiva. Docente e investigador -

Institución Universitaria Esumer,

Medellín, Colombia.

Email: luis.delgado@esumer.edu.co

ORCID: https://orcid.org/0000-0002-3642-3692

Tipología:

Artículo de reflexión

Fecha de recibido: agosto 29 de 2016

Fecha de aceptación: junio 082017

Publicado en línea: noviembre 27 de 2017

Para citar este artículo:

Durango, G. M. y Delgado, V. L. (2017). Diseño metodológico para la estructuración de portafolios de inversión según el perfil de riesgo del inversionista. Clío América, 11(22), 177-187. Doi:

$10.21676 / 23897848.2439$
RESUMEN: El alto nivel de desarrollo cuantitativo que está siendo aplicado en la creación de portafolios de inversión en el mercado financiero, permite desarrollar metodologías basadas en criterios y en modelos matemáticos ajustados a la realidad económica que logre minimizar pérdidas y obtener un mayor beneficio económico esperado por el inversionista. El objetivo del artículo consiste en definir una metodología para la creación de unos portafolios de inversión, según el perfil de riesgo del inversionista para portafolios con múltiples activos y que a través del valor en riesgo permiten simular la optimización del portafolio y generar mayor rentabilidad. Al determinar que el portafolio está compuesto por acciones de diferentes empresas colombianas y en distintos sectores económicos, y a través del monitoreo permite incorporar un análisis de los riesgos financieros a los cuales están expuestos los inversionistas, permitiendo reducir la probabilidad de pérdida que afecte el patrimonio u originada por cambios $\mathrm{y} / 0$ movimientos adversos en los factores de riesgo que afectan el precio 0 valor final.

Palabras clave: portafolio; inversión; perfil de riesgo; límites; rentabilidad.

JEL: G11, G12, G14

ABSTRACT: The high level of quantitative development that is being applied in the creation of investment portfolios in the financial market allows developing methodologies based on criteria and mathematical models adjusted to the economic reality that manages to minimize losses and obtain a greater economic benefit expected by the investor. The objective of the article is to define a methodology for the creation of an investment portfolio, according to the risk profile of the investor for portfolios with multiple assets and that, through value at risk, simulate the optimization of the portfolio and generate greater profitability. When determining that the portfolio is composed of shares of different Colombian companies and in different economic sectors, and through monitoring it allows incorporating an analysis of the financial risks to which investors are exposed, allowing to reduce the probability of loss that affects the equity or caused by changes and / or adverse movements in the risk factors that affect the price or final value.

Keywords: portfolio; investment; risk profile; limits; profitability. 


\section{INTRODUCCIÓN}

El riesgo en los mercados financieros tiene como punto de referencia más importante la crisis del 1929 con el desplome de la bolsa de Nueva York, debido a la sobrevaloración de los precios de sus activos, como consecuencia de la alta especulación que ocurría en el mercado financiero. A partir de este momento las instituciones del mercado financiero comenzaron a tomar conciencia para administrar el riesgo.

El fenómeno de la profunda crisis de 2008 que afectó al mercado financiero mundial, conocido como subprime mortgage crisis ${ }^{1}$, generó profundos descensos en las cotizaciones bursátiles y deterioro de la economía global, causando así un nivel de riesgo de impago $0^{2}$ cada vez mayor. El extremo nivel de aversión al riesgo y el colapso de la acelerada corrección de los activos, hizo que el crecimiento global en términos económicos se disminuyera notablemente.

La gestión del riesgo en las organizaciones ha evolucionado a lo largo de la historia, pasando de ser un factor negativo, aislado y subjetivo a convertirse en una oportunidad, administrado integralmente. En lo particular, la teoría de los mercados financieros ha permitido que los administradores de los portafolios de inversión minimicen los niveles de incertidumbre a los que están expuestos los títulos o conjunto de títulos que allí se transan, con el fin de mejorar su probabilidad de éxito en sus rendimientos (Gumensindo, Torres y Jimenez, 2000).

En la estructuración de los portafolios de inversión se identifican seis tipos de riesgos: riesgo de mercado, crédito y contraparte, liquidez, operacional, legal y reputacional (De Rivera, 2002). También es importante precisar que, de acuerdo con los objetivos del inversionista, en un portafolio se identifican tres perfiles según el riesgo: agresivo, moderado y conservador, cuyas cualidades están determinadas por la edad, nivel de ingresos, liquidez, patrimonio acumulado, obligaciones financieras, conocimiento financiero y nivel de aversión al riesgo (Frenkel, 2003).

1 Hipotecas de alto riesgo.

2 Incumplimiento de pago por parte de un emisor.
El objetivo del artículo es mostrar los resultados de la estructuración de un portafolio de inversión adaptado a las condiciones actuales del mercado local, con una metodología reconocida en la gestión de riesgos (Johnson, 2000), que permita cumplir con los dos postulados básicos: minimizar el riesgo del inversionista y maximizar la rentabilidad, según su perfil de riesgo (De Lara Haro, 2005).

Con el fin de generar una opción al inversionista del mercado de capitales colombiano, se decide crear un portafolio que permite de una manera teórica, práctica y metodológica, realizar la combinación correcta de activos financieros y obtener la mayor eficiencia entre riesgo y rentabilidad, y que según (Mas, 1998) las diferencias entre riesgo y rentabilidad también dependen de los miembros de grupos estratégicos, definidos por variables comunes.

\section{METODOLOGÍA}

Según el objeto de estudio, la investigación de campo intenta comprender y resolver alguna situación, necesidad o problema en un contexto determinado, en cuyo caso compete la generación de un portafolio de inversión para un perfil de riesgo característico, según su nivel de aversión. Este a su vez, trabaja en un ambiente natural en el que conviven las personas y las fuentes consultadas, de las que obtendrán los datos más relevantes a ser analizados. Lo que hace importante el análisis de los portafolios de inversión en el mercado de capitales colombiano.

Para ello se utilizaron herramientas e instrumentos de investigación cuantitativa. Se realizó revisión documental, así como la administración de la rentabilidad esperada, a través del Valor en Riesgo, que como lo expresa (Juseluis, 2006) son modelos econométricos y estadísticos basados en estimaciones de un fenómeno en particular, desde ahora $\mathrm{VaR}$, como medida de riesgo en una cartera de inversiones de activos financieros y que determina posibles resultados negativos en la cartera (Novales, 2014). Teniendo en cuenta la probabilidad y horizonte de tiempo, el VaR demuestra un valor límite de probabilidad de pérdida a precios de mercado en la cartera y asumiendo mercados normales. El diseño metodológico para la estructuración de un portafolios de inversión según el perfil de riesgo del inversionista, se realizó mediante técnicas de 
análisis de contenido como de la revisión documental y la cual supone, (Galeano, 2004) especial énfasis en lo vivencial 0 en la interacción que se establece entre los sujetos de la investigación. En este proceso de investigación, se establece contacto con diferentes actores como inversionistas personas naturales y agentes del sistema financiero, encaminados a generar un modelo que permita eficiencia del sistema.

El diseño del instrumento se realizará bajo la metodología del modelo de regresión lineal a través de un modelo matemático que permita aproximar la relación de dependencia entre una variable dependiente e independiente, con el fin de darle una opción al inversionista del mercado de capitales colombiano. Se desarrollará un portafolio que permitirá de una manera teórica, práctica, metodológica hacer la combinación correcta de activos financieros y obtener la mayor eficiencia entre riesgo y rentabilidad.

Esta metodología admite que los errores en la medición de las variables explicativas sean independientes entre sí, con una línea de tendencia en una serie de datos obtenidos en un período, reconociendo analizar si la variable dependiente ha crecido o decrecido en comparación con las mismas variables de otros modelos.

A continuación, se describe en la figura 1 el conjunto de procedimientos empleados durante la investigación, por medio de un proceso lógico a través del cual se obtiene el conocimiento.

\section{Figura 1.}

Procedimiento para la administración del portafolio.

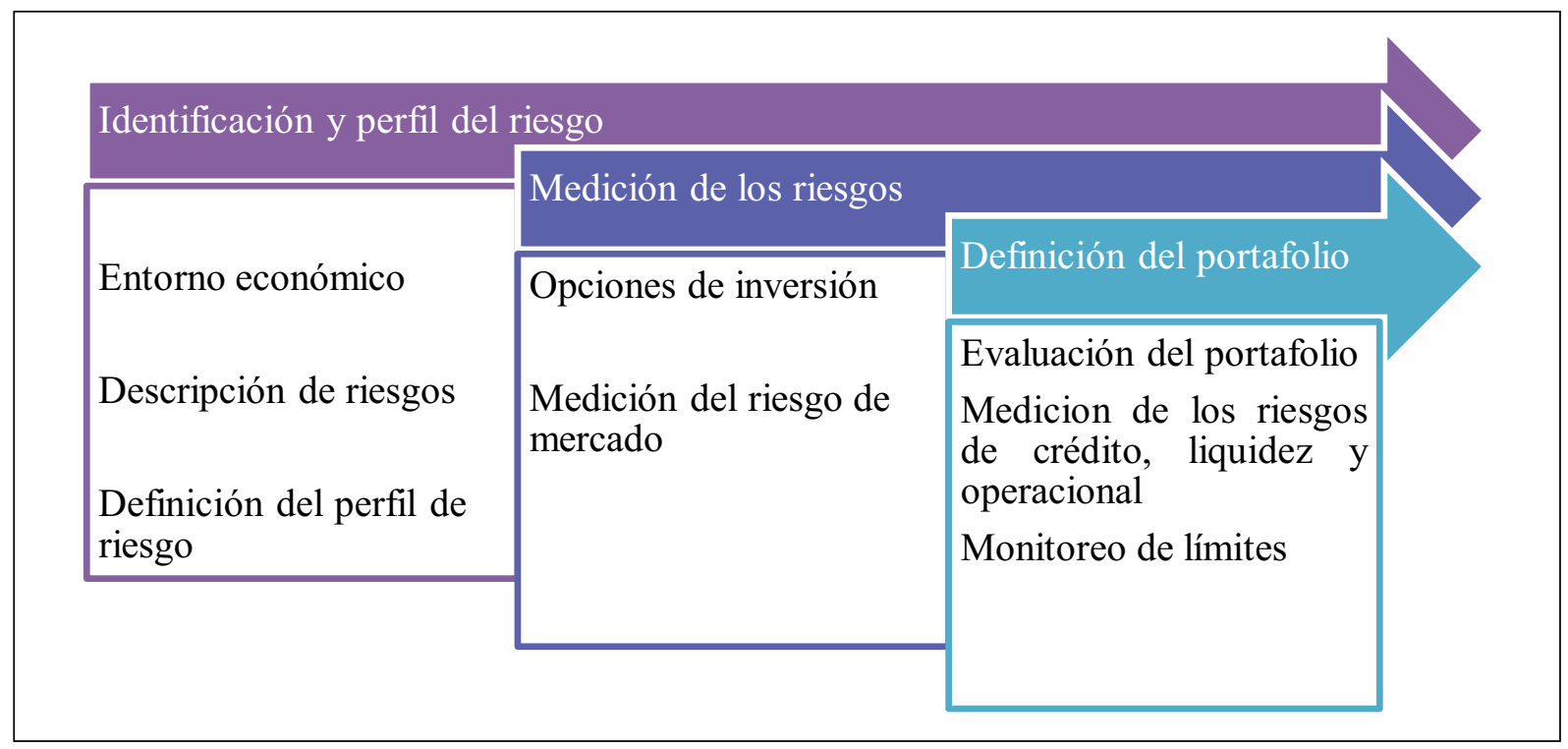

Fuente: elaboración propia.

\section{Identificación y perfiles de riesgo}

El riesgo definido como una amenaza, peligro 0 incertidumbre a la que se ve enfrentado un inversionista, por un evento u acción sea del mercado 0 ajeno a él, en el que este pudiera afectar la situación financiera de su portafolio de manera negativa 0 positiva (Pascale, 1998). Cuando se refiere a una ma- nera positiva es debido a que el riesgo es analizado como una oportunidad, gestionado y administrado bajo marcos comunes y metodologías aceptadas a nivel mundial que permiten que sea un factor de oportunidad (Herrera, 1994).

En la tabla 1 se identifican y describen los tipos de riesgo. 
Diseño metodológico para la estructuración de portafolios de inversión según el perfil de riesgo del inversionista

\section{Tabla 1.}

\section{Relación Causa - Efecto de Riesgos}

\begin{tabular}{|l|l|}
\hline \multicolumn{1}{|c|}{ TIPOS DE RIESGOS } & \multicolumn{1}{|c|}{ CONSECUENCIA PARA EL MERCADO ACCIONARIO } \\
\hline Mercado & $\begin{array}{l}\text { fly to quality }{ }^{3} \text {, (Escobar y Cuartas, 2006) se define como venta de activos ries- } \\
\text { gosos en países emergentes o aun en desarrollados, buscando un mejor nivel de } \\
\text { rentabilidad con un riesgo asociado mucho menor. Los activos de renta fija al } \\
\text { subir las tasas se vuelven más atractivos que el mercado accionario. }\end{array}$ \\
\hline Variación del valor de los flujos de caja de un activo debido a la inflación en tér- \\
minos de poder adquisitivo. Los activos que son de tasa fija están más expuestos \\
a este tipo de riesgo. Las tasas de interés variables tienen una menor exposición \\
a este tipo de riesgo.
\end{tabular}

Fuente: elaboración propia

\section{Perfil del Inversionista}

\section{Perfil conservador:}

Los individuos con esta característica, buscan con su inversión obtener unas ganancias estables y que no pierdan el valor del dinero en el tiempo, por tanto su grado de preferencia es una menor rentabilidad a cambio de minimizar los riesgos que están tomando en su inversión (González, 2008, p. 11).

De acuerdo con González (2008), un ejemplo de una inversión para un perfil conservador es un bono

3 Salida por calidad, técnicamente se conoce como participación de activos riesgosos por el mercado emergente en el que opera. corporativo. Una de las grandes debilidades se presenta en los cambios y movimientos de largo plazo en economías vulnerables, la rentabilidad puede generar pérdida de valor, debido a que rinden por debajo de la inflación.

\section{Perfil moderado:}

Según lo afirma Alas (2016), el inversionista desea obtener en sus portafolios de inversión un equilibrio entre riesgo y rentabilidad. Por su naturaleza, tienden a ser más cautelosos, pero están dispuestos a incurrir en un nivel de aversión al riesgo un poco mayor que el perfil conservador para obtener mejores utilidades y en determinados casos, este tipo 
de inversionistas no le temen a perder un pequeño porcentaje de su capital invertido. Un ejemplo de ello es un portafolio diversificado, en el que se presentan productos de inversión tanto en renta fija como en renta variable, en porcentajes diferenciados; tales como los productos de CDTs ${ }^{4}$ y acciones locales o internacionales. Sin embargo, el porcentaje de renta variable será menor al que escogería un inversionista con perfil arriesgado (p.16).

\section{Perfil arriesgado:}

Son inversionistas que, según su perfil, están dispuestos a tomar grandes riegos para obtener grandes ganancias, pero también tienen una gran posibilidad de incurrir en grandes pérdidas 0 en la totalidad del capital invertido; por tanto, su nivel de cobertura frente al riesgo es bajo. Las personas con este perfil se destacan por tener edades jóvenes, adineradas, solteras y aún sin hijos; con bajo nivel de endeudamiento de largo plazo y prefieren portafolios que combinan renta variable, derivados y moneda extranjera, en un período de corto plazo (Jaramillo, 2009, p. 9, 10).

\section{Definición de variables:}

La meta y el tiempo: los inversionistas, en su mayoría, presentan múltiples metas; sin embargo, no están dispuestos a tomar el mismo nivel de riesgo para todas ellas. Generalmente, una meta crítica 0 un objetivo que tiene un marco de tiempo menor a tres años es lo que dicta un perfil bastanteconservador. Metas menos críticas u objetivos con horizontes mayores a tres años dictan perfiles más agresivos ya que en este caso es posible recuperarse de una baja en el mercado. Adicionalmente, y como lo afirman (Contreras Bronfman y Arenas, 2015) lograr una combinación entre rentabilidad y riesgo es un gran problema a nivel financiero, a ello se le suma la calidad del tiempo.

Personalidad: los fenómenos económicos influyen en la toma de decisión de una inversión. Por tanto, las percepciones, creencias y actitudes adjudican la personalidad del inversionista. Es así como las

4 Certificado de depósito a término. Producto de inversión en renta fija, rentabilidad definida al comienzo de la inversión. características de la personalidad destacan la necesidad de poder, reconocimiento y prestigio que viene acompañado con la obtención de capital (Manquero, 2006).

Edad: Se espera que más personas con mayor edad son menos tolerantes al riesgo y que personas más jóvenes sean más arriesgadas. Lo común en personas mayores de 55 años se consideren conservadoras, entre los $35 y$ 55 años de edad son inversores moderados y menores a 35 años arriesgados (López, 2016).

Ingresos y patrimonio: mientras mayores sean los ingresos y el patrimonio se está dispuesto a tomar mayores riesgos, por lo menos para un subconjunto de inversiones (Punina y Salazar, 2015).

Se resume lo anterior en la figura 2, los niveles de tolerancia al riesgo:

\section{Figura 2.}

Niveles de tolerancia de riesgo.

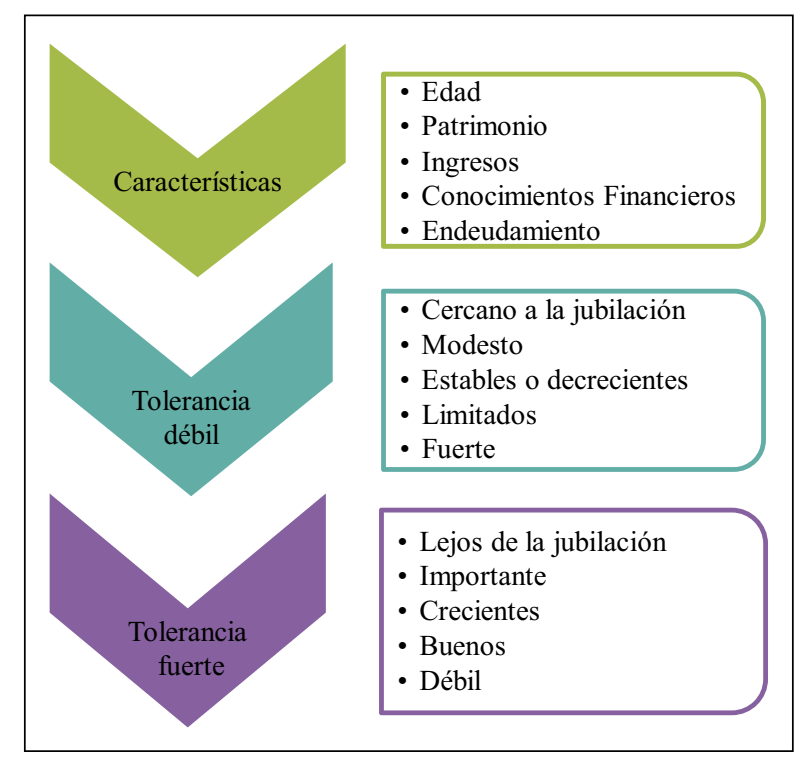

Fuente: elaboración propia.

En la tabla 2, se describe el siguiente instrumento, que define dos encuestas para clasificar al inversionista, basado en variables sociodemográficas como en la personalidad, conocido como el test de preguntas de Kostolany (1985). Así mismo, la figura 3 detalla la valoración de la puntuación para clasificar los perfiles de riesgo. 


\section{Tabla 2.}

\section{Encuesta 1: Clasificación del perfil inversionista}

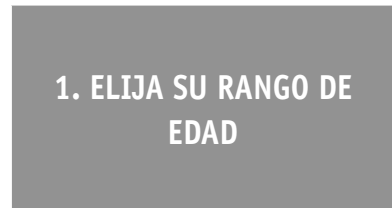

a) Más de 60 años

1

b) 46 a 60 años

2

c) 36 a 45 años

3

d) 26 a 35 años

4

e) Menos de 25 años

5

5. ¿SU PRESUPUESTO

PERSONAL SUFRE

GRANDES VARIACIONES?

a) Totalmente de acuerdo

1

b) De acuerdo

2

c) Neutral

3

d) Desacuerdo

4

e) Totalmente en desacuerdo

5

\section{2. ¿QUÉ PORCENTAJE DE \\ SUS AHORROS ACTUALES DESEA INVERTIR?}

a) $80 \%$ a $100 \%$

1

b) $60 \%$ a $80 \%$

2

c) $40 \%$ a $60 \%$

3

d) $20 \%$ a $40 \%$

4

e) Menos del $20 \%$

5

6. ¿POSEE USTED PÓLIZAS DE SEGUROS DE VIVIENDA, VEHÍCULO, ESTUDIOS PARA SUS HIJOS, ETCÉTERA?

a) Totalmente de acuerdo 5

b) De acuerdo 4

c) Neutral

3

d) Desacuerdo

2

e) Totalmente en desacuerdo 1

\section{3. ¿EN CUÁNTOS AÑOS DESEA EMPEZAR A \\ GASTAR LOS FONDOS INVERTIDOS?}

4. ¿EL PRINCIPAL OBJETIVO DE SU INVERSIÓN ES CUBRIR SUS GASTOS CORRIENTES?

a) 1 año

a) Totalmente de acuerdo

1

b) De acuerdo

b) 2 a 5 años

2

c) Neutral

3

d) Desacuerdo

4

e) Totalmente en desacuerdo

e) Más de 21 años

5

\section{7. ¿A LA HORA DE \\ INVERTIR BUSCA \\ INVERSIONES RENTABLES O SEGURAS?}

a) Muy seguras

a) Muy estable 1

5

b) Seguras

b) Estable

2

c) Balanceadas

4

c) Neutral 3

3

d) Rentables

d) Inestable

4

e) Muy rentables

2

e) Muy inestable

1

Fuente: elaboración propia. 


\section{Figura 3.}

\section{Puntuación de perfiles.}

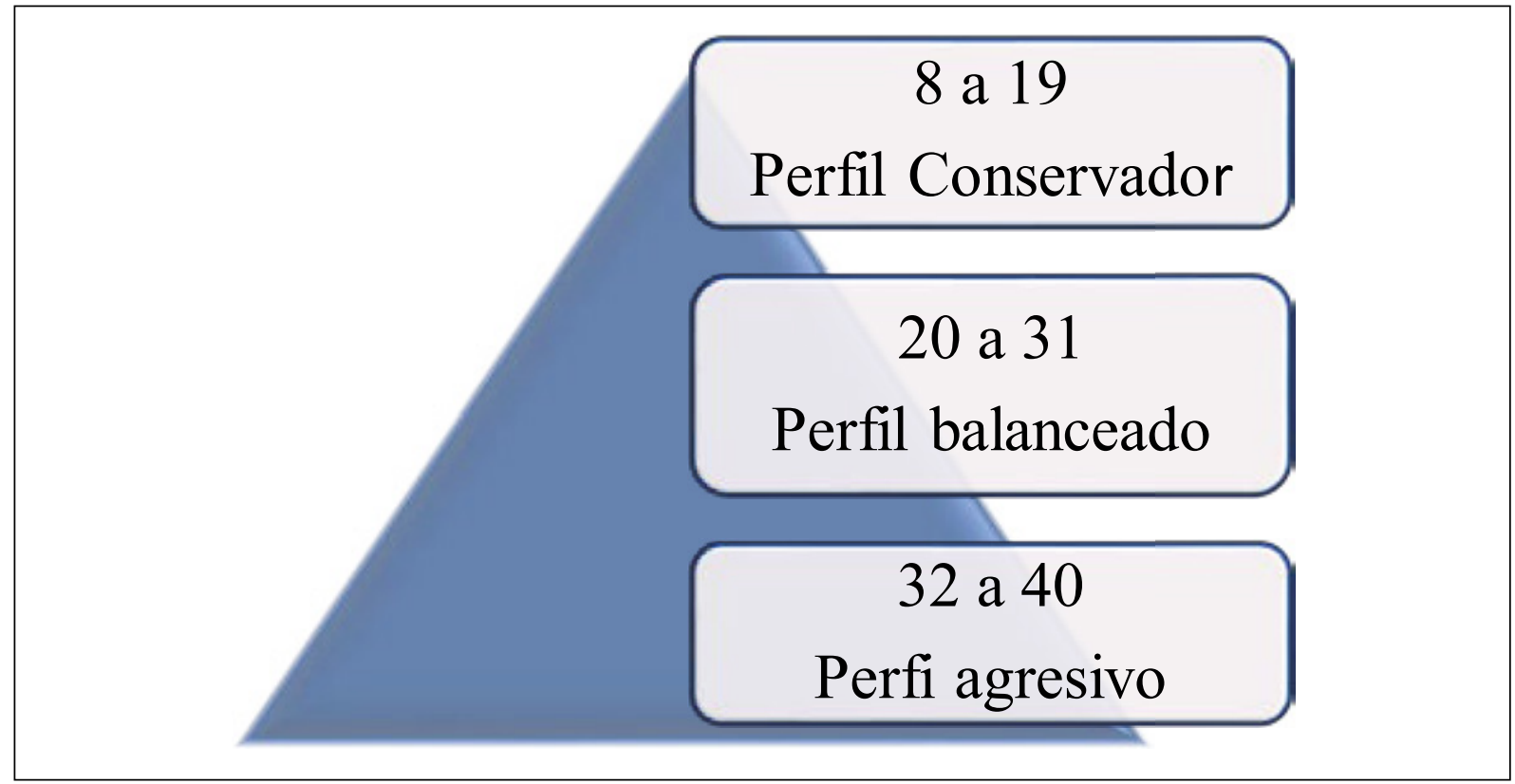

Fuente: elaboración propia.

A su vez, el Test Kostolany determina el grado de riesgo que el encuestado está dispuesto a aceptar en una inversión; son 15 preguntas realizadas desde la subjetividad del autor André Kostolany y no son generalizables a todos los casos; tal como lo ilustra la tabla 3.

De acuerdo con el test, el número de respuestas a y $b$ determinan el nivel de aversión. Siendo (b) un perfil inversionista con mayor aversión al riesgo y (a) con menor aversión.

\section{Medición de los riesgos}

Variables dependientes: las opciones que se escogieron para el perfil de riesgo agresivo arrojado, de acuerdo con el instrumento, fueron acciones listadas en la Bolsa de Valores de Colombia (BVC). Tales como Grupo Sura, Conconcreto, Ecopetrol, Preferencial Bancolombia, Bancolombia, Nutresa, Celsia, Inversiones Argos, Cementos Argos, ISA, Éxito y BVC; teniendo en cuenta las siguientes variables: bursatilidad ${ }^{5}$, sector económico, liquidez, capitalización bursátil, solidez de la compañía, expectativas de crecimiento y valorización, con el fin de optimizar el rendimiento del portafolio y controlar el valor en riesgo del mismo.

5 Bursatilidad: indicador de desempeño que mide el comportamiento de los últimos seis meses de las acciones con mayor grado de liquidez. Definidas en alta, media, baja y mínima. 
Diseño metodológico para la estructuración de portafolios de inversión según el perfil de riesgo del inversionista

\section{Tabla 3.}

\section{Encuesta 2: Test de Kostolany}

\begin{tabular}{|c|c|c|c|c|c|c|}
\hline $\begin{array}{l}\text { 1. ¿HACE } \\
\text { PLANES } \\
\text { PARA LAS } \\
\text { VACACIO- } \\
\text { NES? }\end{array}$ & $\begin{array}{l}\text { 2. LA SEÑAL } \\
\text { DE TRÁFICO } \\
\text { ESTÁ EN ROJO, } \\
\text { PERO NO } \\
\text { LLEGA NINGÚN } \\
\text { AUTOMÓVIL. }\end{array}$ & $\begin{array}{l}\text { 3. ESTÁ } \\
\text { USTED } \\
\text { DE BUEN } \\
\text { HUMOR }\end{array}$ & $\begin{array}{l}\text { 4. EN SU PLATO } \\
\text { HAY UN EXCEP- } \\
\text { CIONAL BUEN } \\
\text { BOCADO. }\end{array}$ & $\begin{array}{l}\text { 5. ELIGE USTED } \\
\text { CUADROS } \\
\text { FUNDAMENTAL- } \\
\text { MENTE }\end{array}$ & $\begin{array}{l}\text { 6. SI VISI- } \\
\text { TA UN PAÍS } \\
\text { EXTRANJERO } \\
\text { POR PRIMERA } \\
\text { VEZ }\end{array}$ & $\begin{array}{l}\text { 7. TRAE USTED } \\
\text { DE PARÍS A SU } \\
\text { ESPOSA }\end{array}$ \\
\hline $\begin{array}{l}\text { a. Con varios } \\
\text { meses de } \\
\text { anterioridad. }\end{array}$ & $\begin{array}{l}\text { a. Espera usted } \\
\text { el color verde }\end{array}$ & $\begin{array}{l}\text { a. Por la } \\
\text { mañana }\end{array}$ & $\begin{array}{l}\text { a. Lo deja } \\
\text { para el final }\end{array}$ & $\begin{array}{l}\text { a. Por su valor } \\
\text { como inversión }\end{array}$ & $\begin{array}{l}\text { a. Va a un } \\
\text { restaurante } \\
\text { que le han } \\
\text { recomendado }\end{array}$ & $\begin{array}{l}\text { a. El perfume } \\
\text { favorito } \\
\text { encargado }\end{array}$ \\
\hline $\begin{array}{l}\text { b. Poco } \\
\text { antes de que } \\
\text { comiencen. }\end{array}$ & $\begin{array}{l}\text { b. Prosigue la } \\
\text { marcha en rojo. }\end{array}$ & $\begin{array}{l}\text { b. Mejor por } \\
\text { la tarde. }\end{array}$ & $\begin{array}{l}\text { b. Comienza con el } \\
\text { mismo. }\end{array}$ & $\begin{array}{l}\text { b. Por su propio } \\
\text { gusto. }\end{array}$ & $\begin{array}{l}\text { b. Va a uno } \\
\text { desconocido } \\
\text { del lugar }\end{array}$ & $\begin{array}{l}\text { b. Una nueva } \\
\text { marca que es } \\
\text { muy "in" y } \\
\text { que a usted le } \\
\text { gusta. }\end{array}$ \\
\hline $\begin{array}{l}\text { 8. CUANDO } \\
\text { USTED ELIGE } \\
\text { PLAZA EN } \\
\text { EL AVIÓN, } \\
\text { ELIGE UNA } \\
\text { POR }\end{array}$ & $\begin{array}{l}\text { 9. ¿QUÉ } \\
\text { JUEGA EN LA } \\
\text { RULETA (O QUÉ } \\
\text { JUGARÍA SÍ LE } \\
\text { LLEVARAN)? }\end{array}$ & $\begin{array}{c}10 . \\
\text { CUANDO } \\
\text { BUSCA UNA } \\
\text { VIVIENDA } \\
\text { SE FIJA MÁS } \\
\text { EN: }\end{array}$ & $\begin{array}{l}\text { 11. ¿QUÉ HARÍA } \\
\text { SI CAMINO DE } \\
\text { IR A VER UNA } \\
\text { PELÍCULA DE } \\
\text { CRİMENES SE } \\
\text { ENTERA USTED } \\
\text { DE QUIÉN ES EL } \\
\text { ASESINO? }\end{array}$ & $\begin{array}{c}\text { 12. SU } \\
\text { EMPRESA } \\
\text { CAMBIA DE } \\
\text { RADICACIÓN. } \\
\text { TIENE } \\
\text { USTED QUE } \\
\text { TRASLADARSE } \\
\text { A OTRA CIUDAD }\end{array}$ & $\begin{array}{l}\text { 13. ¿QUÉ } \\
\text { QUIERE QUE } \\
\text { HAGA SU } \\
\text { ESPOSA? }\end{array}$ & $\begin{array}{l}\text { 14. ¿QUÉ } \\
\text { POSTURA ES } \\
\text { MEJOR PARA } \\
\text { LA VIDA } \\
\text { FAMILIAR? }\end{array}$ \\
\hline $\begin{array}{l}\text { a. Seguridad } \\
\text { y comodidad }\end{array}$ & $\begin{array}{l}\text { a. Oportunidades } \\
\text { iguales }\end{array}$ & $\begin{array}{l}\text { a. El aspecto } \\
\text { de ésta }\end{array}$ & $\begin{array}{l}\text { a. Iría a pesar } \\
\text { de ello a ver la } \\
\text { película }\end{array}$ & $\begin{array}{l}\text { a. Le molesta } \\
\text { fundamental- } \\
\text { mente }\end{array}$ & $\begin{array}{l}\text { a. Permanecer } \\
\text { en casa } \\
\text { haciendo sus } \\
\text { labores }\end{array}$ & $\begin{array}{l}\text { a. El hombre } \\
\text { que cuida del } \\
\text { presupuesto y } \\
\text { la mujer que es } \\
\text { más prodiga }\end{array}$ \\
\hline $\begin{array}{l}\text { b. El } \\
\text { vecino/a. }\end{array}$ & $\begin{array}{l}\text { b. Todo a un } \\
\text { número. }\end{array}$ & $\begin{array}{l}\text { b. En el } \\
\text { entorno. }\end{array}$ & $\begin{array}{l}\text { b. Prescindiría de } \\
\text { ello. }\end{array}$ & $\begin{array}{l}\text { b. Espera alegre } \\
\text { el traslado. }\end{array}$ & b. Que trabaje. & $\begin{array}{l}\text { b. El hombre } \\
\text { que tiene mano } \\
\text { ligera con el } \\
\text { dinero mientras } \\
\text { la mujer cuida } \\
\text { del ahorro }\end{array}$ \\
\hline
\end{tabular}

Fuente: (Kostolany, 1987)

Rentabilidad del portafolio: con el fin de observar el funcionamiento del modelo, se asigna una rentabilidad esperada igual en todos los activos, al calcular la matriz de covariancias y correr las macros que optimizan el modelo bajo las restricciones he- chas al mismo (rentabilidades negativas mayores a 0 , ponderación del activo en el portafolio iguales 0 mayores a 0 , y teniendo un VaR del $10 \%$ ) se pueden observar en la tabla 4 los siguientes resultados del VaR del modelo. 
Tabla 4.

Modelo 1 y 2 para un Portafolio de Inversión

\begin{tabular}{|c|c|c|c|c|c|c|}
\hline \multirow{2}{*}{ MODELO 1} & VARIANZA & VaR DIARIA & Wi & Wi & $\operatorname{Re}^{6}$ & VaR \\
\hline & $\%$ & $\%$ & $\$$ & $\%$ & $\%$ & Deseada \\
\hline ECOPETROL & 1,06 & 2 & 99754062 & 10 & 15 & \\
\hline PFBCOLOM & 1,23 & 2 & 5101954 & 1 & 15 & \\
\hline BCOLOMBIA & 1,18 & 2 & 21250425 & 2 & 15 & \\
\hline NUTRESA & 1,03 & 2 & 132403133 & 13 & 15 & $\begin{array}{l}100 \%: \\
7,67 \%\end{array}$ \\
\hline CEMARGOS & 1,2 & 3 & 5489182 & 1 & 15 & \\
\hline ISA & 1,05 & 3 & 105696606 & 11 & 15 & \\
\hline ÉXITO & 1,21 & 3 & 84085235 & 8 & 15 & \\
\hline BVC & 1,38 & 2 & 57395027 & 5 & 15 & \\
\hline \multicolumn{3}{|c|}{ ASIGNACIÓN TOTAL DE RECURSOS } & 511175625 & 51 & & \\
\hline \multirow{2}{*}{ MODELO 2} & VARIANZA & $\begin{array}{c}\text { VaR DIARIA } \\
\text { ACTIVO } \\
\end{array}$ & Wi & Wi & $\operatorname{Re}$ & VaR \\
\hline & $\%$ & $\%$ & $\$$ & $\%$ & $\%$ & Deseada \\
\hline CONCONCRETO & 2,95 & 5 & 11565640 & 1 & 3 & \\
\hline ECOPETROL & 1,06 & 2 & 276678485 & 28 & 3 & \\
\hline NUTRESA & 1,03 & 2 & 143432742 & 14 & 22 & $\begin{array}{l}100 \%: \\
11,81 \%\end{array}$ \\
\hline \multicolumn{3}{|c|}{ ASIGNACIÓN TOTAL DE RECURSOS } & 431676868 & 43 & & \\
\hline \multicolumn{2}{|l|}{ Modelo 1 y 2} & $\%$ & $\$$ & & & \\
\hline \multicolumn{2}{|c|}{ Variación diario portafolio } & 63 & 6299471 & & & \\
\hline \multicolumn{2}{|c|}{ Variación semanal portafolio } & 41 & 14086045 & & & \\
\hline \multicolumn{2}{|c|}{ Variación mensual portafolio } & 2 & 28172090 & & & \\
\hline \multicolumn{2}{|c|}{ Variación Anual portafolio } & 10 & 100000998 & & & \\
\hline
\end{tabular}

Fuente: elaboración propia

La segunda simulación del modelo con todos los activos tiene la siguiente variación, puesto que la rentabilidad esperada de los activos fue estimada con variación en pesos de los últimos cinco años de cada activo; tal como se observa en la tabla 4.

\section{RESULTADOS}

Con el fin de observar el VaR del $10 \%$ del portafolio para optimizar el modelo con las restricciones del mismo, no hace una locación de todos los recursos en los activos, debido a que la varianza hace que el VaR fuese superado por dicha razón; por tanto, el modelo no asigna todos los recursos. Si en el modelo se aumenta el VaR del portafolio a un $60 \%$ se observa que se realiza una asignación de todos los recursos en los activos señalados por el modelo 1 en su optimización.

De acuerdo con el análisis del portafolio en donde dos activos financieros son sustraídos, es decir la acción del Éxito y la acción BVC, se observa que el

6 Rentabilidad esperada. 
VaR no varía, sino la asignación de los recursos, y de esta manera la decisión de invertir es o no la más adecuada.

Se puede observar en los resultados que hay una asignación menor de recursos frente al modelo 1 , esto debido a que las rentabilidades esperadas de ciertos activos son menores que en el primer modelo. Los activos con una mayor volatilidad presentan mayores rentabilidades, lo que tiene como consecuencia una mayor asignación en estos activos, pero implícitamente un mayor riesgo; por tanto, el modelo suaviza la asignación de dichos recursos y así mantener el VaR en la restricción del $10 \%$.

En la última simulación del modelo, y teniendo en cuenta la exclusión de los dos activos y las rentabilidades histórica esperadas iguales a las del segundo modelo, se observa una reducción en los montos de los recursos a asignar, teniendo en cuenta que lo más importante es analizar la optimización que hace el portafolio es concentrarse en activos de alta rentabilidad y bajas varianzas.

\section{CONCLUSIÓN}

Por medio del monitoreo se valora la calidad del desempeño del modelo de medición del riesgo definido, así como el cumplimiento y eficiencia de los límites establecidos. Este monitoreo permite reconocer de forma oportuna las deficiencias de la administración del riesgo y genera una retroalimentación efectiva en las operaciones futuras de inversión.

La estrategia de monitoreo consiste en evaluar a través del modelo valor en riesgo (VaR) la probabilidad de pérdida potencial en el portafolio. Este monitoreo se deber realizar diariamente, con el fin de recomponer el portafolio de forma oportuna para mitigar riesgos, puesto que al determinar que el portafolio está compuesto por acciones de diferentes empresas, este incorpora un análisis de los riesgos financieros de las empresas, con el fin de conocer las perspectivas de las empresas.

El control permanente de los límites en cada uno de los tipos de riesgo permite tomar medidas adecuadas y oportunas que reduzcan al mínimo la probabilidad de sufrir una pérdida considerable que afecte el patrimonio del inversionista.
La cuantificación del riesgo de crédito como incertidumbre para el inversionista y para la empresa se ve afectado por el tipo de empresa, medido este por el nivel de solidez y estructura financiera, donde se tomaron empresas sólidas y con respaldo patrimonial.

El riesgo de contraparte no existe puesto que se desconoce la contraparte en la negociación de los activos y por ende este riesgo se mitiga.

Por otro lado, el riesgo de incumplimiento por parte del emisor no existe, puesto que se desconoce la contraparte en la negociación de los activos y por ende este riesgo se mitiga. El riesgo de liquidez medido por el tipo de acciones y su nivel de versatilidad, donde se escogieron acciones de alta bursatilidad con el fin de minimizar una posible iliquidez en los activos financieros.

De la misma manera, el riesgo operacional es asumido por la firma comisionista de bolsa y se ve afectado por factores internos en la operación del negocio, el cual es asumido por la empresa disminuyendo este riesgo para el inversionista.

\section{REFERENCIAS BIBLIOGRÁFICAS}

Alas, R. J. J. (2016). Riesgo y rentabilidad de las inversiones en la bolsa de valores (Tesis doctoral). Universidad de El Salvador, San Salvador.

Contreras, O. E., Bronfman, R. S. y Arenas, C. E. V. (2015). Estrategia de inversión optimizando la relación rentabilidad-riesgo: evidencia en el mercado accionario colombiano. Estudios Gerenciales, 31(137), 383-392.

De Lara Haro, A. (2005). Medición y control de riesgos financieros. España: Editorial Limusa.

De Rivera, J. I. P. S. (2002). La gestión de riesgos financieros de mercado y crédito. España: Pearson.

Escobar, H. y Cuartas, V. (2006). Diccionario Económico y Financiero 3ra Edición. Colombia: Sello Editorial Universidad de Medellín.

Frenkel, R. (2003). Globalización y crisis financieras en América Latina. Revista de la CEPAL, 80, 41-54.

González, R. C. A. (2008). Portafolios y Estrategias de Inversión (Doctoral dissertation). Universidad del Salvador, El Salvador.

Gumensindo, R., Torres, J. J. y Jimenez, J. I. (2000). La Gestión del Riesgo Financiero. España: Piramide. 
Herrera, A. 0. (1994). Las nuevas tecnologías y el futuro de América Latina: riesgo y oportunidad. Siglo XXI. México: Editorial de la universidad de Naciones Unidas.

Jaramillo, A. (. (2009). Propuesta para la implementación de nuevos productos con derivados financieros en el mercado colombiano (Tesis de pregrado). Recuperado de: http://www.bdigital. unal.edu.co/2477/1/3396727.2009.pdf

Johnson, C. A. (2000). Métodos de evaluación del riesgo para portafolios de inversión (No. 67). Chile: Banco Central. Recuperado de http://si2.bcentral.cl/public/ pdf/documentos-trabajo/pdf/dtbc67.pdf

Juselius, K. (Ed.). (2006). The cointegrated VAR model: methodology and applications. Oxford, Reino Unido: 0xford University press.

Kostolany, A. (1985). El fabuloso mundo del dinero y la bolsa. España: Planeta.

Kostolany, A. (1987). Estrategia bursátil: la mejor guía para inversores y especuladores. España: Planeta.
López, G. J. C. (2016). Las finanzas personales, un concepto que va más allá de su estructura. Estado del arte período 2006-2016 (Tesis de maestría). Universidad EAFIT, Colombia.

Manquero, L. E. R. (2006). Factores subyacentes de cultura y personalidad empresarial que influyen en la intención de invertir. EconoQuantum, Revista de Economia y Negocios, 3(1), 63-89.

Mas, R. F. J. (1998). Relación rentabilidad-riesgo en el contexto de los grupos estratégicos. Revista Europea de Dirección y Economía de la Empresa, 7(1), 33-46.

Novales, A. (2014). Valor en Riesgo. Madrid, España: Universidad Complutense.

Pascale, R. P. (1998). Decisiones financieras. Tercera Edición. Buenos Aires: Editorial Macchi.

Punina, G. J. y Salazar, M. C. (2015). El riesgo de crédito y la liquidez Didáctica Editorial Nuevo Milenio (Tesis de pregrado). Universidad Técnica de Ambato, Ecuador. 